\title{
Cervical Footprint Anthropometry in Indian Population: Implications on Design of Artificial Disc Replacement Devices
}

\author{
Arvind Gopalrao Kulkarni, Vishwanath Mahabaleshwar Patil, \\ Shashidhar Kantharajanna Bangalore, Abhishek Saraf \\ Mumbai Spine Scoliosis and Disc Replacement Centre, Bombay Hospital, Mumbai, India
}

\section{Study Design: Cross-sectional study.}

Purpose: To accurately measure the dimensions of cervical endplates based on computed tomography (CT) scans in Indian population and assess accuracy of match with currently available cervical disc prostheses.

Overview of Literature: The dimensions of currently available cervical disc replacement implants are based on early published geometrical measurements of vertebrae endplates for Caucasian population. To author's knowledge, similar study has not been published for patients from Indian subcontinent.

Methods: CT scans of cervical spine of patients from Indian subcontinent were collected and reviewed. Seventy patients (54 men and 16 women; aged 18-56 years with average of 37 years) who underwent CT scans of cervical spine were included in study. 3D CT scans of sub axial cervical spine (C3 to C7) were analyzed. The anterior-posterior (AP) and central mediolateral (CML) dimensions of superior and inferior endplates from $\mathrm{C} 3$ to $\mathrm{C} 7$ were measured using digital measuring system.

Results: A total of 560 endplates of 70 patients were included in the study. The AP diameter of cervical endplates ranged from 0.87 to $2.47 \mathrm{~cm}$. The CML diameters ranged from 0.84 to $2.98 \mathrm{~cm}$. For levels C3/C4 and C4/C5 for AP dimension Prestige-LP (90.5\%) and Prodisc-C (89\%) discs showed higher percentage of matching than Discover discs (58.5\%). For CML diameter, Prestige-LP (69.5\%), Prodisc-C (70\%) and Discover (39.5\%) discs showed almost similar matching with measured endplates. For levels C5/C6 and C6/C7 for AP dimension, Prestige-LP (67.25\%), Prodisc-C (49.35\%) and Discover (51.5\%) discs showed similar matching. For CML diameter Prestige-LP (32\%), Prodisc-C (27.5\%) and Discover (42.2\%) discs showed poor matching with measured endplates.

Conclusions: This study indicates need for redesign of cervical disc prostheses to match Indian patients. The collected anthropometric dimensions from this study may be used to design and develop indigenous artificial total disc replacement prosthesis and even cervical cages in India. With the present study being a small pilot study, the authors recommend anthropometric CT measurements in larger number of Indian patients in order to validate footprint dimensions for designing better-matched prosthesis.

Keywords: Cervical spine footprint; Endplate diameter; Cervical total disc replacement; Cervical disc herniation; Cervical myelopathy

Received May 18, 2015; Revised Jun 14, 2015; Accepted Jun 17, 2015

Corresponding author: Arvind Gopalrao Kulkarni

Mumbai Spine Scoliosis and Disc Replacement Centre (MSSDRC), Bombay Hospital,

New Marine Lines, Mumbai 400020, India

Tel: +91-9892875490, Fax: +91-22-22080871, E-mail: drarvindspines@gmail.com 


\section{Introduction}

With the evolution of motion preservation spine surgery, cervical total disc replacement (C-TDR) has become an increasingly popular modality for treatment of cervical degenerative disc disease and has been suggested as an alternative to fusion [1-3]. Disc prostheses for replacement are available in various sizes of endplates. Several studies have elaborated the importance of congruence of implant surface and endplate geometry [4,5]. Inadequate load distribution across peripheral apophyseal ring of vertebral endplates, presumably caused by asymmetric implantation and implant under-sizing, has been proposed as one of the contributing factors for subsidence, the most frequent device related problem of C-TDR. It has also been shown that the cortical shell of the vertebra provides $45 \%-75 \%$ of resistance to axial load, and disc prosthesis should closely match the size of the endplate in order to prevent subsidence and failure. Mismatch of endplate dimensions between the patient's anatomy and implant design can lead to inadequate load distribution associated with failure of device design. Therefore, the prostheses should have a footprint, matching the endplate that it is replacing to have the best results for dissipating the load evenly rather than only in concentrated areas.

Currently the most common available types of C-TDR in India are the Prestige-LP (Medtronics, U.S-2007), Discover (DePuy, Raynham, MA, USA) and Prodisc-C (Synthes, West Chester, PA, USA) with around 600 procedures performed in a year (as per market sources). The dimensions of the footprints of C-TDR prosthesis currently available in India are based on published geometrical measurements of cadaveric studies [6] or plain radiography studies [7] in the Western population. Previous morphometric related studies conducted in Indian subcontinent have showed considerable differences in measurements of anatomy such as odontoid and dorso-lumbar pedicle morphometry between Indians and Caucasians [8,9]. A recent study showed that currently available lumbar disc prostheses are much smaller than lumbar endplates regarding anteriorposterior (AP) and central mediolateral (CML) diameter. A study by Thaler et al. [10] has shown considerable mismatch between human values obtained by computed tomography and footprints of C-TDR prostheses available in the market. Study of foot plate geometric features of Indian patients could contribute to design a best fit cervical disc suited for Indians as they contribute to $17 \%$ of world population with approximately one Indian in every five individuals worldwide and $32.9 \%$ of Asia's population [11]. Considering the variable anatomy between different ethnic groups, use of implants designed by Western cadaveric studies in Indian population cannot be recommended. With this background, the aim of the current study was to measure endplates of cervical spine in Indian patients using computed tomography (CT) scans and digital measurement tools, and assess the matching qualities of currently available cervical disc prostheses. Comparisons of skeletal geometric features between race and ethnic groups may yield insights about the mechanisms of prosthesis that could contribute to design a best fit implant among Indians.

\section{Materials and Methods}

A total of 560 endplates in 70 patients (54 men and 16 women; aged 18 to 56 years; average age, 37 years) were assessed. For CT scans, Siemens SOMATOM Sensation Cardiac (Siemens Medical Solutions, Forchheim, Germany) 64-slice CT was used with $120 \mathrm{kV}, 250 \mathrm{mAs}$, rotation 0.95 , and $1-\mathrm{mm}$ slice thickness with $3 \mathrm{D}$ reconstruction of images. Scan coverage of vertebral levels was from $\mathrm{C} 1$ to D1 in apparently normal spine. The images were stored with picture archiving communication system. Then the digital measuring tools were used to measure distances after adjusting the axis parallel and perpendicular at each of the endplate on sagittal images, just making sure that there was no obliquity. Four measurements were taken for each endplate as described by Thaler et al. [10]: the AP diameter, the mediolateral diameters at two locations (ML1/2) and the CML diameter as illustrated in Fig. 1. First, the AP diameter was divided into three parts. Lines perpendicular to the AP diameter were drawn between the anterior and the middle thirds (ML1), and between the middle and the posterior thirds (ML2). Then the AP diameter was divided into two parts. A line perpendicular to the AP diameter was drawn between the anterior and posterior halves (CML). All measurements excluded the uncinate process.

To determine matching sizes of the endplate we compared vertebral endplate diameters with dimensions of most frequently used prostheses available in India at the time of study (Prestige-LP, Discover, Prodisc-C). The vertebral levels were organized into two groups: group I covering the levels $\mathrm{C} 3 / \mathrm{C} 4$ and $\mathrm{C} 4 / \mathrm{C} 5$ and group II cover- 


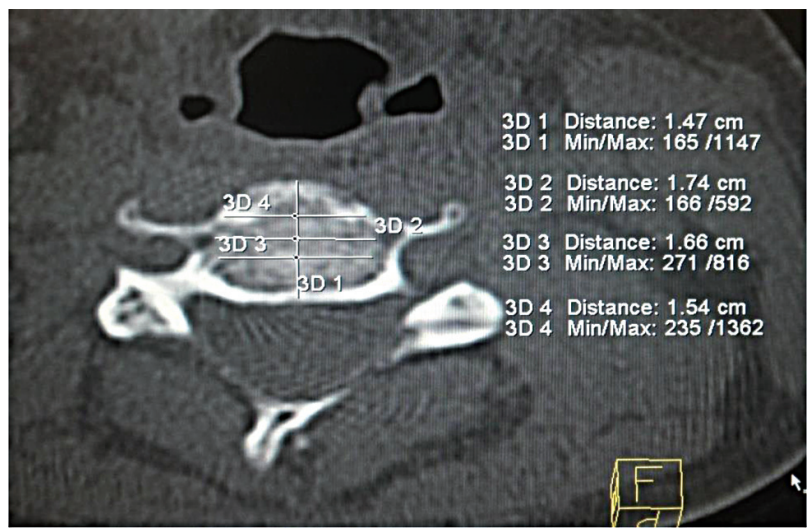

Fig. 1. Measurement of vertebral diameters, in the axial computed tomography (CT) slide of the upper endplate of. AP, anterior-posterior dimension (3D1); CML, center mediolateral diameter (3D2-measured between the anterior and posterior halves of the AP diameter, perpendicular to the AP diameter); ML2, mediolateral diameter 2 (3D3measured between the middle and posterior thirds of the AP diameter, perpendicular to the AP diameter); and ML1, mediolateral diameter 1 (3D4, measured between the anterior and middle thirds of the AP diameter, perpendicular to the AP diameter).

ing the levels C5/C6 and C6/C7-because of the majority of surgical procedures are performed at the levels C5/C6 and C6/C7. Data were processed using the Statistical Package for Social Sciences (SPSS Ver. 20, IBM CO., Armonk, NY, USA). Figures were created with Excel (Excel 2007, Microsoft, Redmond, WA, USA).

\section{Results}

The mean values of AP, ML1, ML2, and CML diameters of the upper and the lower endplates from $\mathrm{C} 3$ to $\mathrm{C} 7$ are shown in Table 1. The AP diameter of cervical endplates ranged between 0.87 to $2.47 \mathrm{~cm}$. The smallest AP diam- eter of the available prostheses were $1.2,1.42$, and $1.2 \mathrm{~cm}$ for Prodisc-C, Discover and Prestige-LP, respectively. The largest AP diameter of available prostheses was 1.8, 1.87, and $1.8 \mathrm{~cm}$ for Prodisc-C, Discover and Prestige-LP prostheses, respectively. The CML diameters ranged from 0.84 to $2.98 \mathrm{~cm}$. The smallest CML diameter of available prostheses were 1.5, 1.42, and $1.2 \mathrm{~cm}$ for Prodisc-C, Discover and Prestige LP, respectively. The largest CML diameter of available prostheses was 1.7, 1.87 and $1.6 \mathrm{~cm}$ for Prodisc, Discover and Prestige-LP prostheses, respectively

Regarding AP diameter in group I, Prestige-LP and Prodisc-C discs showed a higher percentage of matching close to $90 \%$ and Discover discs showing $58 \%$ of matching. For CML diameter, Prestige-LP and Prodisc-C discs showed matching of $69.5 \%$ and $70 \%$, respectively, whereas Discover discs matched in $39.5 \%$ of anatomic endplates.

In group II, Discover discs matched better than the other two counterparts with $51.5 \%$ and $42.2 \%$ matching in AP and CML diameter, respectively, whereas PrestigeLP and Prodisc-C discs matched close to $50 \%$ in AP and $30 \%$ in CML diameter. The detailed percentage matching is illustrated in Table 2.

The graphical representation of percentage matching in group I and II with respect to available diameter is shown in Figs. 2-5.

\section{Discussion}

The biomechanical and anatomical differences in parts of the cervical vertebra have to be given consideration when designing a best fit implant [12]. The posterior and the lateral aspect of the vertebral endplates have been shown to be relatively stronger than the anterior and middle parts

Table 1. Dimension of superior and inferior endplates of cervical vertebrae:In mm

\begin{tabular}{lllll} 
Dimensions & \multicolumn{1}{c}{ AP } & ML 1 & ML 2 & CML \\
C3 inferior & $1.36(1.1-1.74)$ & $1.37(1.04-1.7)$ & $1.404(0.92-1.91)$ & $1.528(1.10-2.13)$ \\
C4 superior & $1.46(0.94-1.87)$ & $1.51(0.75-1.90)$ & $1.48(0.73-2.23)$ & $1.57(0.84-2.24)$ \\
\hline C4 inferior & $1.59(1-1.99)$ & $1.58(1.17-1.9)$ & $1.60(1.19-2.34)$ & $1.69(1.11-2.19)$ \\
C5 superior & $1.58(1.08-1.9)$ & $1.62(0.94-1.8)$ & $1.61(0.75-1.9)$ & $1.80(1.02-2.42)$ \\
\hline C5 inferior & $1.51(1.05-1.83)$ & $1.62(1.20-1.9)$ & $1.57(1.14-1.88)$ & $1.73(1.20-2.98)$ \\
C6 superior & $1.42(0.87-1.94)$ & $1.54(0.87-1.98)$ & $1.63(1.08-2.08)$ & $1.83(1.20-2.82)$ \\
C6 inferior & $1.57(0.88-1.20)$ & $1.68(0.76-2.32)$ & $1.67(1.23-2.26)$ & $1.88(1.41-2.8)$ \\
C7 superior & $1.74(1.2-2.47)$ & $1.96(1.2-2.7)$ & $1.96(1.16-2.5)$ & $2.092(1.3-2.81)$ \\
\hline
\end{tabular}

$\mathrm{AP}$, anterior-posterior; $\mathrm{ML}$, mediolateral; CML, center mediolateral. 
Table 2. Percentage matching of the available cervical disc arthroplasty dimensions with dimensions of the endplates

\begin{tabular}{lllll} 
& AP $(\%)$ & ML1 $(\%)$ & ML2 $(\%)$ & \\
\hline Group 1: C3-C5 & & & & \\
\hline Prestige-LP & 90.5 & 54.8 & 56.1 & 69.5 \\
\hline Prodisc-C & 89.0 & 57.3 & 62.6 & 70.25 \\
\hline Discover & 58.5 & 47.0 & 46.7 & 39.5 \\
\hline Group 2: C5-C7 & & & 34.9 & 32.0 \\
\hline Prestige-LP & 67.25 & 33.3 & 36.8 & 27.5 \\
\hline Prodisc-C & 49.35 & 32.7 & 48.5 & 42.2 \\
\hline Discover & 51.5 & 47.5 & & \\
\hline
\end{tabular}

$\mathrm{AP}$, anterior-posterior; $\mathrm{ML}$, mediolateral; $\mathrm{CML}$, center mediolateral.

\section{Frequency}

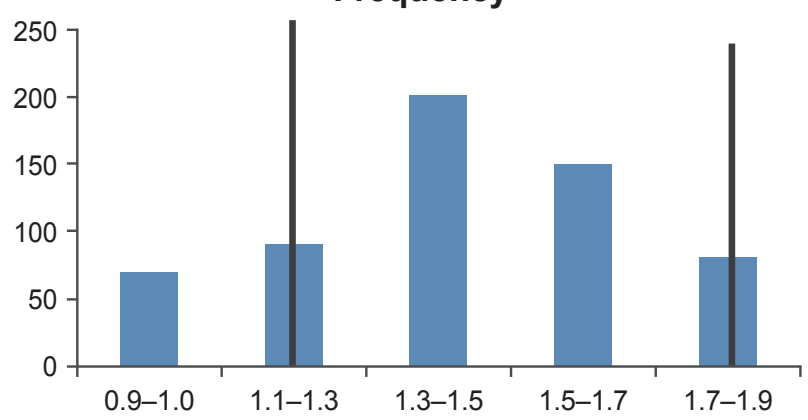

Fig. 2. Anterior-posterior (AP) dimensions of disc at levels C3/C4 and C4/C5 (group I). Comparison of vertebral endplates and available disc prosthesis sizes. Black lines mark AP dimenions of available footprints of cervical disc prostheses.

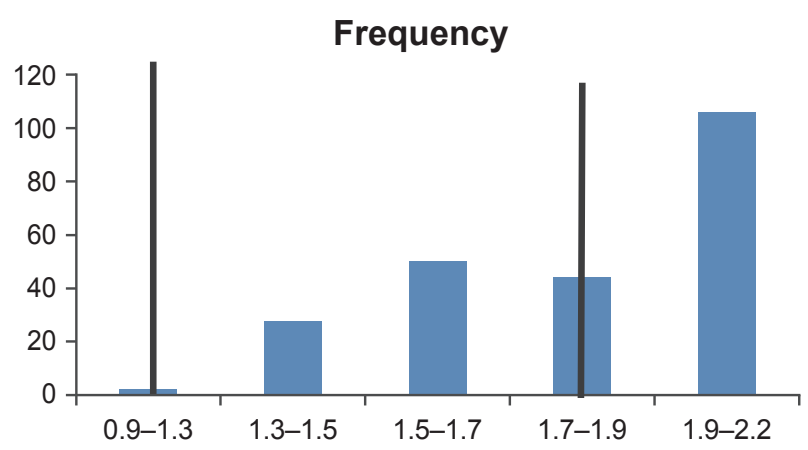

Fig. 3. Center mediolateral dimensions of disc at levels C3/ C4 and C4/C5 (group I). Comparison of vertebral endplates and available disc prosthesis sizes. Black lines mark mediolateral diameters of available footprints of cervical disc prostheses.

[13]. For an implant to effectively transmit the load it has to correspond to these geometrical variations. Link et al. [14] suggested using a rectangular footprint size in cervical arthroplasty in order to cover as much as possible of the cervical endplate, because bending forces are much

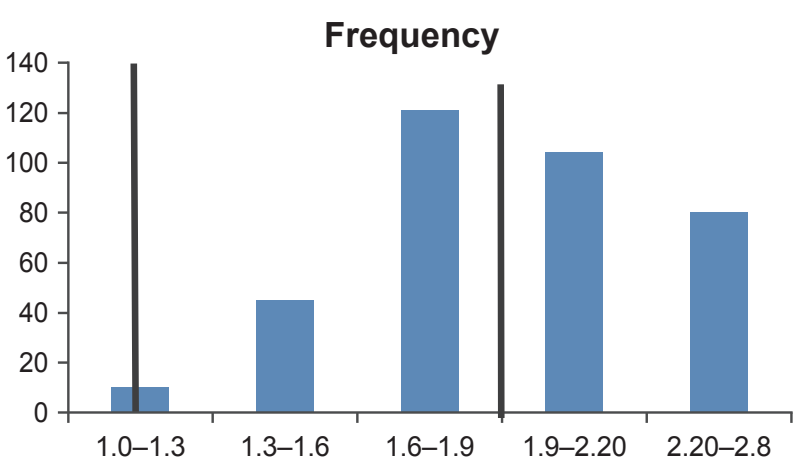

Fig. 4. Anterior-posterior (AP) dimensions of disc at levels C5/C6 and C6/C7 (group II). Comparison of vertebral endplates and available disc prosthesis sizes. Black lines mark AP diameters of available footprints of cervical disc prostheses.

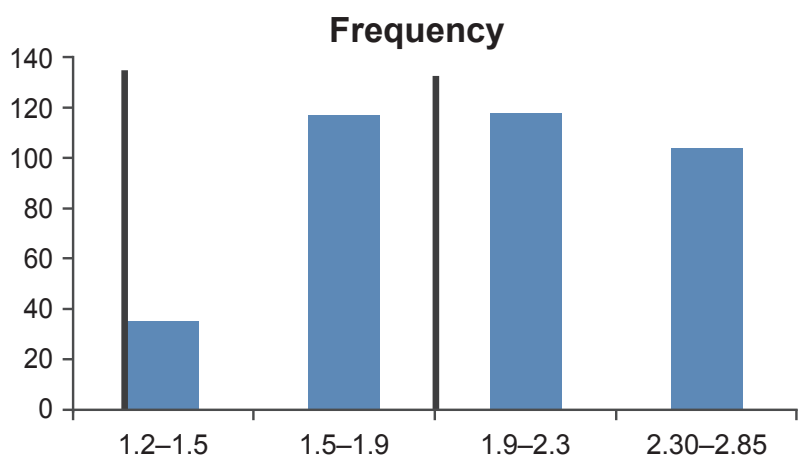

Fig. 5. Center mediolateral dimensions of disc at levels C5/C6 and C6/ C7 (group II). Comparison of vertebral endplates and available disc prosthesis sizes. Black lines mark mediolateral dimensions of available footprints of cervical disc prostheses.

stronger in the lateral aspect of cervical vertebrae than in thoracic or lumbar vertebrae. This is attributable to the greater range of lateral flexion in the cervical spine. For this reason, it has been proposed that the C-TDR device should have as large a footprint as possible to transfer 
Table 3. Dimensions of available implants and cadaveric study by Panjabi et al. [6]

\begin{tabular}{lcccccc} 
Dimensions & Panjabi et al. [6] & Prodisc-C & Discover & Prestige-LP & Thaler et al. [10] & Current study \\
Width/MLD & 16.5 to 22.5 & 15 to 17 & 14.2 to 18.7 & 12 to 16 & 5.6 to 35.7 & 9.2 to 26.8 \\
& & $(15,17,19)$ & $(14.2,15.7,17.2,18.7)$ & $(12,14,16)$ & 12 to 18 & 10.2 to 23.8 \\
Depth/AP & 14.5 to 19.2 & 12 to 18 & 13.7 to 16.7 & 9.6 to 24.7 \\
& & $(12,14,16,18)$ & $(13.7,15.2,16.7)$ & $(12,14,16,18)$ & & \\
\hline
\end{tabular}

MLD, mediolateral diameter; $A P$, anterior-posterior.

weight from the center of the endplate to its stronger periphery. Such ideal foot prints have been shown to improve the biomechanical loads and decrease the incidence of subsidence. Subsidence can result in loss of motion at the implanted disc with increased stress at the adjacent levels due to loss of sagittal balance. Proper sizing of the prosthetic components are crucial to the success and longterm survival of C-TDR. Due to the large variability of the anthropometry of different populations of the world, ethnic groups having a smaller build, such as Indians, are likely to develop technical errors in C-TDR with most of the commercially available prostheses due to the nonavailability of proper sized implants.

The design of the available implants is based on anthropometric measurements of the cadavers [6] and photographs of radiographs [7]. CT evaluation of bony structures is more accurate, and it has been recently found to be useful in determination of lumbar [15] and cervical endplates [10]. Thaler et al. [10] measured the dimensions of cervical vertebrae from computed tomography (CT) scans in 24 patients to assess the accuracy of match achieved with the most common available cervical disc prostheses: Bryan (Medtronic), Prestige-LP (Medtronic), Discover (Depuy), and Prodisc-C (Synthes). A total of 192 endplates in 24 patients (56.3 years) were assessed. Overall, $53.5 \%$ of the largest device footprints were smaller in the AP diameter and $51.1 \%$ in the CML diameter were smaller than cervical endplate diameters. For levels C5/ $\mathrm{C} 6$ and $\mathrm{C6} / \mathrm{C} 7$ an inappropriate size match was noted in $61.9 \%$ as calculated from the AP diameter. Mismatch at the CML diameter was noted in $56.8 \%$. The study found the measured endplates to be significantly larger in comparison to the available foot prints. In contrast in our study we have found that the measured values were both smaller and larger than the available foot prints (Table 3).

The mean values of AP, ML1, ML2, and CML diameters of the upper and the lower endplates from $\mathrm{C} 3$ to $\mathrm{C} 7$ are shown in Table 1. The AP diameter of cervical endplates ranged from 0.96 to $2.47 \mathrm{~cm}$. The smallest AP diameter of the available prostheses were 1.2, 1.42 and 1.2 for ProdiscC, Discover and Prestige-LP, respectively. The largest AP diameter of available prostheses was 1.8, 1.87, and 1.8 $\mathrm{cm}$ for Prodisc-C, Discover and Prestige-LP prostheses, respectively. In group I for all measured dimensions Prestige-LP (69.5\% to $90 \%)$ and Prodisc-C (70.5\% to $89 \%)$ showed higher percentage of matching than Discover discs $(39.5 \%$ to $58.5 \%)$. This can be attributed to the fact that the smallest available AP diameter with Discover was $1.42 \mathrm{~cm}$ and it was larger than more than 50 percent of the measured dimensions (in range of 1.1 to $1.4 \mathrm{~cm}$ ) as seen in Figs. 2 and 3. The CML diameters ranged from 0.92 to $2.68 \mathrm{~cm}$. The smallest CML diameter of available prostheses was 1.5, 1.42, and $1.2 \mathrm{~cm}$ for Prodisc-C, Discover and Prestige-LP discs, respectively. The largest CML diameter of available prostheses was 1.7, 1.87, and $1.6 \mathrm{~cm}$ for Prodisc-C, Discover and Prestige-LP prostheses, respectively. In group II Discover ( $42.2 \%$ to $51.5 \%$ ) showed a marginally better matching than Prestige-LP (32\% to $67.2 \%$ ) and Prodisc-C (27.5\% to $49.35 \%)$ prostheses. This lack of significant matching was attributable to large number of values being more than $1.6 \mathrm{~cm}$ (Figs. 4, 5).

The center of rotation in total disc arthroplasty is also an important biomechanical factor. In cervical disc arthroplasty the center of rotation depends on prosthesis type and movement. Axial position of the center of rotation influences spine flexibility in all loading conditions and the facet force in extension, lateral bending and axial rotation. The anteroposterior position was found to influence spinal flexibility in flexion and extension and the facet force in lateral bending and axial rotation, while the lateral position was not significant.

Proper positioning of the artificial disc during surgery, in particular in the anteroposterior direction, was found to be of critical importance. The footprint mismatches found in the present study largely cause prostheses to be more centrally positioned in the intervertebral space. Po- 
tential consequences of such a discrepancy are subsidence, migration, wrong load distribution affecting the facet joints, loss of sagittal balance and heterotopic ossification due to insufficient range of motion of the functional spinal unit [10].

The dimensions of the available disc prostheses have been designed based on the available literature on anthropometric measurements of endplates. These dimensions and implants based on them are summarized in Table 3 . These reports are based on small Caucasian populations. Application of implants based on these data to Indian population is inaccurate. Studies in the white population, based on CT based evaluation have found large discrepancies in dimensions of lumbar and cervical endplates. In our study, none of the available prosthesis matched all the measured endplate dimensions. The footprint mismatches found in the present study demonstrate the need for designing smaller foot print discs for group I and slightly larger foot prints for the group II. With the widespread application of the C-TDR procedure, it is imperative that population specific discs be designed to avoid potential complications of subsidence, migration, wrong load distribution etc.

This study has other implications as well. In cervical disc fusion surgeries, the success depends on using appropriate implants which match the dimensions of the cervical endplates. The cages which we routinely use in fusion surgeries are either imported implants or the Indian implants, the sizes of which are based on the morphometric measurements of Caucasian population. We recommend that the morphometric measurements done in the present study be extrapolated in re-designing cervical cages.

\section{Conclusions}

There are significant differences in the dimensions of the endplates of Indian patients and the available implants in the market. Under sizing the prosthetic device may lead to subsidence, loosening, heterotopic ossification and biomechanical failure caused by an incorrect center of rotation and load distribution, affecting the facet joints. This study indicates a need for a redesign of the cervical disc prostheses to match the Indian population. As the present study is a pilot study, the authors recommend anthropometric CT measurements in a larger Indian population to validate footprint dimensions designing better-matched prosthesis.

\section{Conflict of Interest}

No potential conflict of interest relevant to this article was reported.

\section{References}

1. Mummaneni PV, Burkus JK, Haid RW, Traynelis VC, Zdeblick TA. Clinical and radiographic analysis of cervical disc arthroplasty compared with allograft fusion: a randomized controlled clinical trial. J Neurosurg Spine 2007;6:198-209.

2. Qureshi SA, Koehler SM, Lu Y, Cho S, Hecht AC. Utilization trends of cervical artificial disc replacement during the FDA investigational device exemption clinical trials compared to anterior cervical fusion. J Clin Neurosci 2013;20:1723-6.

3. Lu Y, McAnany SJ, Hecht AC, Cho SK, Qureshi SA. Utilization trends of cervical artificial disc replacement after FDA approval compared with anterior cervical fusion: adoption of new technology. Spine (Phila Pa 1976) 2014;39:249-55.

4. Lin CY, Kang H, Rouleau JP, Hollister SJ, Marca FL. Stress analysis of the interface between cervical vertebrae end plates and the Bryan, Prestige LP, and ProDisc-C cervical disc prostheses: an in vivo imagebased finite element study. Spine (Phila Pa 1976) 2009;34:1554-60.

5. Kang H, Park P, La Marca F, Hollister SJ, Lin CY. Analysis of load sharing on uncovertebral and facet joints at the C5-6 level with implantation of the Bryan, Prestige LP, or ProDisc-C cervical disc prosthesis: an in vivo image-based finite element study. Neurosurg Focus 2010;28:E9.

6. Panjabi MM, Duranceau J, Goel V, Oxland T, Takata K. Cervical human vertebrae: quantitative three-dimensional anatomy of the middle and lower regions. Spine (Phila Pa 1976) 1991;16:861-9.

7. Gilad I, Nissan M. A study of vertebra and disc geometric relations of the human cervical and lumbar spine. Spine (Phila Pa 1976) 1986;11:154-7.

8. Kulkarni AG, Shah SM, Marwah RA, Hanagandi PB, Talwar IR. CT based evaluation of odontoid morphology in the Indian population. Indian J Orthop 2013;47:250-4.

9. Chadha M, Balain B, Maini L, Dhaon BK. Pedicle morphology of the lower thoracic, lumbar, and S1 
vertebrae: an Indian perspective. Spine (Phila Pa 1976) 2003;28:744-9.

10. Thaler M, Hartmann S, Gstottner M, Lechner R, Gabl M, Bach C. Footprint mismatch in total cervical disc arthroplasty. Eur Spine J 2013;22:759-65.

11. Wikipedia. Demographics of India: Population based study (2014) [Ineternet]. Wikipedia; 2014 [cited 2014 Dec 22]. Available from: https://en.wikipedia.org/ wiki/Demographics_of_India_(2014).

12. Kim SW, Shin JH, Arbatin JJ, Park MS, Chung YK, McAfee PC. Effects of a cervical disc prosthesis on maintaining sagittal alignment of the functional spi- nal unit and overall sagittal balance of the cervical spine. Eur Spine J 2008;17:20-9.

13. Cheng CC, Ordway NR, Zhang X, Lu YM, Fang H, Fayyazi AH. Loss of cervical endplate integrity following minimal surface preparation. Spine (Phila $\mathrm{Pa}$ 1976) 2007;32:1852-5.

14. Link HD, McAfee PC, Pimenta L. Choosing a cervical disc replacement. Spine J 2004;4(6 Suppl):294S$302 \mathrm{~S}$.

15. Gstoettner M, Heider D, Liebensteiner M, Bach CM. Footprint mismatch in lumbar total disc arthroplasty. Eur Spine J 2008;17:1470-5. 